\title{
Early defibrillation in out-of-hospital sudden cardiac death: an Australian experience
}

\author{
I. A. SCOTT \& G. J. FITZGERALD
}

Ipswich General Hospital, Ipswich, Queensland and Queensland Ambulance Services Board

\section{SUMMARY}

All patients with primary cardiac disease presenting with out-of-hospital sudden cardiac death (OH-SCD) to a provincial hospital were reviewed retrospectively over a 5-year period from 1985 to 1989 . This coincided with the introduction of out-of-hospital defibrillation (OH-DEFIB) by ambulance officers. Of 215 patients, $17(9 \%)$ survived to leave hospital alive, 15 of whom underwent OH-DEFIB. There was an increase in survivors from $4 \%$, prior to OH-DEFIB, to $9 \%$ of all cardiac arrests, but this was not statistically significant $(P=0.3)$. However, long term survival amongst immediate survivors was associated with a statistically significant improvement following the introduction of OH-DEFIB (15 of $30(50 \%)$ vs. 2 of $19(10.5 \%), P<0.01)$.

Mean call-out, at-scene and transfer times did not significantly vary between survivors and non-survivors. A total of 155 (72\%) had a known cardiac history, with the majority $(74 \%)$ of arrests occurring at home. Of 134 witmessed arrests, only $46(34 \%)$ underwent bystander-initiated cardioplumonary resuscitation (CPR).

A programme in CPR aimed at relatives of known cardias patients, and the adoption of a paramedic protocol which improves oxygenation at the time of arrest are recommended.

\section{INTRODUCTION}

In the 20 years since Pantridge \& Gettes (1967) introduced a mobile intensive care unit (MICU) in Belfast, U.K., the principle of pre-hospital coronary care has become part of established emergency medical systems in Australia and elsewhere.

Correspondence: Dr I. Scott, Ipswich General Hospital, Ipswich, Queensland, Australia. 
Many operational models apply to pre-hospital coronary care, with procedures and skills varying from CPR with oxygen, to full paramedic protocols including intubation and drug administration. Eisenberg et al. (1990) have identified five $\stackrel{\mathbb{\Omega}}{\stackrel{2}{\circ}}$ categories of response: three single responses - emergency medical technicians $\underset{\Rightarrow}{\Rightarrow}$ (EMT), EMT with defibrillation (EMT-DEFIB) and paramedics - as well as two combined systems, EMT/paramedics and EMT-DEFIB/paramedics. Survival of patients from OH-SCD varies from 2 to $26 \%$, both within, and between, the various models.

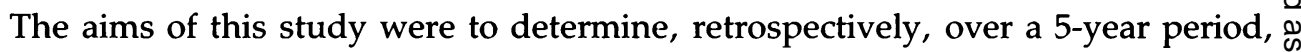
the incidence and patterns of $\mathrm{OH}-\mathrm{SCD}$ within a provincial city environment, the $\overrightarrow{-}$ effectiveness of ambulance initiated OH-DEFIB and the prevalence of bystanderinitiated CPR.

\section{METHODS}

OH-SCD was defined as sudden loss of cardiac output treated by the Ipswich centre of the Queensland Ambulance Transport Brigade (QATB) and transported $\stackrel{\odot}{\odot}$ to Ipswich General Hospital between 1 January 1985 and 31 December 1989. All $\overrightarrow{0}$ patients in whom arrest was caused by intrinsic cardiac disease, and in whor resuscitation efforts were attempted up to the time of arrival at hospital, wete included in the study. Patients with non-cardiac causes of circulatory failure (e.g. respiratory failure, trauma, CNS failure, metabolic or electrolyte disorders, toxin, drug or terminal illness), were excluded, as were patients where resuscitation was not attempted or was terminated on site by a medical practitioner.

Ipswich General Hospital is a 360 acute bed public hospital serving the city of Ipswich and surrounding West Moreton Shires (population approximately 85000) located $40 \mathrm{~km}$ southwest of Brisbane. The hospital has the only emergency department and intensive care unit in the area. In October of 1986, in accordance with a statewide policy to introduce OH-DEFIB, 12 officers from Ipswich Ambulance Centre undertook a 2-week course in this technique, so as to provide, on a $24-\mathrm{h}-\mathrm{a}-\mathrm{3}$ day basis, certified officers within a three-man response team. The course involved instruction and assessment in advanced resuscitation, rhythm recognition and decision making. The ambulance service initially used Lifepack 5 (physio control) and subsequently Hewlett-Packard (HP 43120A Hewlett-Packard Medical Instruments Group, Andorex, MA, U.S.A.) portable defibrillators.

Ambulance officers were permitted to cardiovert ventricular fibrillation (VF) or ? ventricular tachycardia (VT) if in cardiac arrest. If in another rhythm they were ${ }_{0}$ required to continue CPR and transport the patient to hospital. The protocols $\mathrm{\omega}$ permitted a maximum of three shocks on any single episode. However, if a change of rhythm was effected then the protocol could reconmence with any subsequent episode of treatable rhythm.

Data was analysed using D-base 3 (Ashton-Tate) and statistical analysis used chi-square for comparison of discrete variables and a two-tailed Student's $t$-test for continous variables. $P<0.05$ was deemed statistically significant. 


\section{RESULTS}

Over the 5-year period, 1985 to 1989, 215 patients were identified as fulfilling entry criteria, with a steady increase in the annual number of OH-SCD patients from 25 in 1985 to 60 in 1989 (Fig. 1).

Patients were mostly men $(72 \%)$ with a mean age of 64.3 years $(\mathrm{SD} \pm 12.4$ years) and a range of 25-94 years. A total of 169 (78.6\%) had ischaemic heart disease as the cause of arrest. Of 187 patients where prior medical history was obtained, 155 (72\% of the total group) had a known past cardiac history. The majority of arrests $(74 \% ; n=159)$ occurred at home, with no particular predilection to either time of day, or day of the week.

In October 1986, of 215 patients, 48 preceded, and 167 followed the introduction, of continuous cardiac monitoring and OH-DEFIB. A total of 103 of the monitored patients $(62 \%)$ were defibrillated by ambulance officers with an average of three shocks (range 1-15) $92(55 \%)$ and $5(3 \%)$ had VF and VT respectively as rhythms on ambulance arrival.

Survivors were classed as: (1) immediate survivors: those who had a circulation restored and were admitted to hospital from the emergency department and; (2) long-term survivors: those who were subsequently discharged alive from hospital.

There were 54 immediate survivors accounting for $25 \%$ of all cardiac arrests. This percentage fell after the introduction of OH-DEFIB from 39.6 to $29 \%$ of the defibrillated group $(P<0.02)$ although the yearly number of immediate survivors rose slightly. Seventeen long-term survivors were identified $(8 \%$ of the total group), of whom 15 underwent defibrillation (Fig. 2), representing $15 \%$ of all defibrillated patients, and constituting a rise in survival from 4 to $9 \%$ of all arrests $(P=0.3)$ (Table 1$)$.

The number of immediate survivors who survived long-term amongst defibrillated patients was significantly greater than in non-monitored patients (15 out of 30 [50\%] vs. 2 out of 19 [10.5\%], $P<0.01)$.

The mean ambulance call-out time for all patients was $5.3 \mathrm{~min}$ which did not vary significantly between survivors and non-survivors. Similarly the on-scene time for all patients was $13.3 \mathrm{~min}$ although this figure was $11.5 \mathrm{~min}$ for those who were defibrillated.

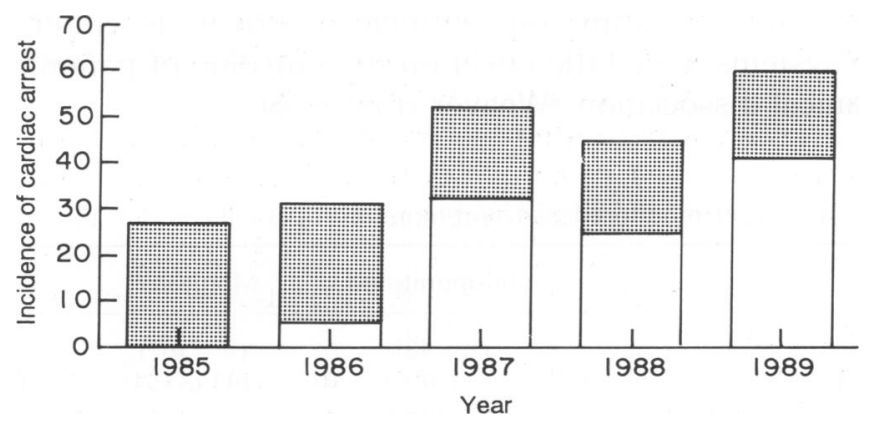

Fig. 1 Ipswich sudden cardiac death survey. Yearly incidence of cardiac arrests, $n=215$. 


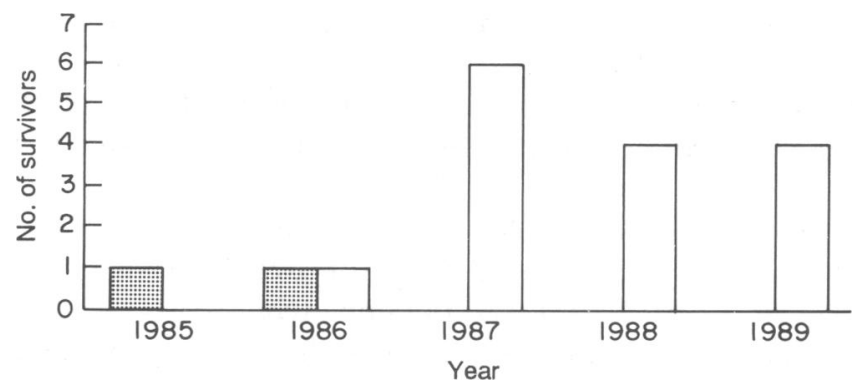

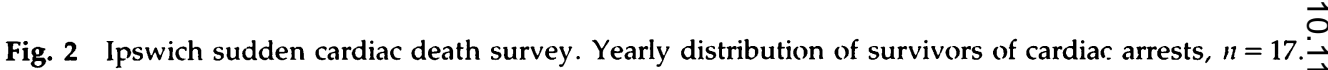

CPR was initiated by bystanders in $55(26 \%)$ of the 215 patients. Of $134 \mathrm{n}$ 'tnessedarrests (representing $62.3 \%$ of the total group) $46(34 \%)$ had CPR initiated, compared to nine $(11 \%)$ of unwitnessed arrests. Of 54 immediate survivors, $18(33 \%) \vec{\circ}$

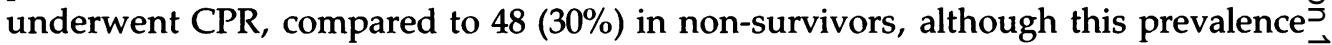
was higher (11 out of 17 [65\%]) in the group of long-term survivors. It should bez noted that no accurate information was available as to the time interval between the arrest and initiation of bystander CPR.

Sex and mean age did not vary significantly between long-term survivors and $\vec{\oplus}$ non-survivors, although immediate survivors, as a group, tended to be older the non-survivors (mean age 67.2 years vs. 63.2 years; $P=0.05$ ). All monitored long term survivors had either VF or VT on ambulance arrival, and were discharged ass functionally independent (although detailed neuropsychological testing was not performed).

\section{DISCUSSION}

Cardiac arrest may be defined as the sudden cessation of cardiac output which is uniformly fatal in the absence of medical intervention. With the immediate response of trained personnel and definitive care, an increasing proportion of patients suffering OH-SCD may survive. Improvement in mortality, however, is confined to those arrests where rhythm, on ambulance arrival, is either VF or VT emergency care systems have little effect on the outcome of patients with asystole or electromechanical dissociation (Weaver et al., 1986).

Table 1. Survival of monitored and non-monitored patients

\begin{tabular}{lccc}
\hline & Non-monitored & Monitored & Total group \\
\hline & $(n=48)$ & $(n=167)$ & $(n=215)$ \\
DEFIB no. (\%) & 0 & $103(61.7)$ & $103(47.0)$ \\
Immed. survivors no. (\%) & $19(39.6)$ & $35(21.0)$ & $54(25.0)$ \\
Long term survivors no. $(\%)$ & $2(4.2)$ & $15(9.0)$ & $17(7.9)$ \\
\hline
\end{tabular}


Numerous studies, using multivariate analysis, have concluded that early initiation of CPR and defibrillation are the strongest predictive variables for longterm survival (Eisenberg et al., 1979; Crampton, 1984; Weaver, et al. 1984). In one study (Eisenberg et al., 1979) CPR administered within $4 \mathrm{~min}$ of an arrest, combined with defibrillation in $8 \mathrm{~min}$, yielded a $25 \%$ survival. Older age was also weakly associated with survival, as supported by this study.

In a controlled, randomized trial of early OH-DEFIB administered by EMTs in witnessed arrests with VF, compared to basic EMT care only, early defibrillation was significantly associated with a better outcome $(42 \%$ survival vs. $19 \%$ respectively) (Eisenberg et al., 1984). All patients received CPR.

In another study Stults et al. (1984) compared ambulance OH-DEFIB with CPR only in a rural setting, and demonstrated significantly better long-term survival (14 vs. $4 \%$ ) in the former group, although early defibrillation had no effect on the number of immediate survivors. Indeed, in our study, there was a statistically significant decrease in the number of immediate survivors following the introduction of OH-DEFIB.

This is difficult to explain given that ambulance response times, prevalence, duration of CPR and patient characteristics were similar in both groups.

Data relating to out-of-hospital resuscitation of arrest patients in this country are scarce. Sammel et al. (1981) reported that, of 434 patients suffering out-ofhospital VF and resuscitated by paramedic teams in the Sydney metropolitan area during 1979 , 35\% were immediate survivors and $21 \%$ long-term survivors - an impressive figure given a mean delay of $15.9 \mathrm{~min}$ before the arrival of paramedics and defibrillation. However, $80 \%$ of patients received CPR from bystanders, standard ambulance teams or medical practitioners, although no information was available regarding the timing of CPR. The use of paramedic protocols (endotracheal intubations, intravenous drug administration and defibrillation) may explain the better outcomes in this study compared to the present one, despite Ipswich ambulance times being considerably shorter (mean call-out time of $5 \mathrm{~min}$ ). The low incidence of bystander-initiated CPR was probably another factor mitigating against better outcomes.

A recent update of the Sydney paramedic experience revealed that mean call-out time has improved to $7.2 \mathrm{~min}$, although mean at-scene time remains at $29 \mathrm{~min}$ (Hall et al., 1989). Of 362 patients with $\mathrm{OH}-\mathrm{SCD}$ attended during a 3-month period to October 1988 only $48 \%$ received some form of CPR within 3 min after collapse, with $21 \%$ immediate survival respectively in patients with VF and asystole - no long-term survival figures were given.

A recent study from Perth, W. A. (Jacob \& Oxer, 1990) reported long-term survival in $17.3 \%$ (40 of 231 ) of patients with VF treated with a defibrillation only protocol, a figure comparable with ours.

In the period covered by our study there was a significant increase in the number of cardiac arrests reported by ambulance and hospital services, for which there may be a number of reasons. Firstly, more patients who were previously considered dead on ambulance arrival now have resuscitation attempted because of enthusiastic trained personnel. Secondly, greater public awareness of the potential for successful intervention may have prompted a call for the ambulance, rather than the family doctor, in the first instance. Finally, a real increase in the incidence 
Table 2. Long term survival - comparative analysis

\begin{tabular}{lrc}
\hline & \% of all arrests & $\%$ of DEFIB rhythms \\
\hline Eisenberg et al. (3) $(n=21$ 937) & & \\
EMT & 4.9 & 11.4 \\
EMT-D & 8.0 & 14.8 \\
Paramedic & 9.6 & 17.9 \\
EMT/paramedic & 13.2 & 24.7 \\
EMT-D/paramedic & 17.6 & 30.0 \\
Sydney $(10)(n=434)$ & - & 21.0 \\
Ipswich $(n=215)$ & 9.0 & 14.8 \\
\hline
\end{tabular}

of SCD cannot be excluded, possibly reflecting improved survival from previous acute coronary events such as myocardial infarction (as a result of better medical treatments), following which patients arrest out-of-hospital.

Eisenberg et al. (1990) has reviewed pre-hospital coronary care in 29 U.S.A. cities and found the survival rate ranging from 2 to $29 \%$. The differential effect on? survival of bystander CPR, early defibrillation and paramedic protocols with inclusion criteria similar to those used for this study, may be assessed by com paring outcomes between the different operation models, as shown in Table $2 . \cdot \vec{\cdot}$ Assuming the Ipswich QATB system equates with an EMT-DEFIB system, ouke survival rate following defibrillation of $9 \%$ compares favourably with the meann rate of $8 \%$ for EMT-DEFIB.

In conclusion, ambulance OH-DEFIB in a provincial setting, with rapid response times, significantly increased the number of immediate survivors. Comparing oun experience with that of metropolitan centres using paramedic protocols, but where $\stackrel{\mathbb{Q}}{\circ}$ call-out and at-scene times are longer, there is reason to expect that survival may be improved further. As a further priority, training for the public in CPR should $B$ be promoted, particularly in relation to families with members at risk of SCD章 given that most arrests occur at home in patients with a known cardiac history.

\section{ACKNOWLEDGEMENTS}

We wish to acknowledge the assistance of officers of the Ipswich QATB Ambulance Centre for providing data on patient resuscitation, to the General Practitioners of Ipswich City for providing information pertaining to past medical history, to Din Stewart Parkinson for assisting in prepartion of diagrams, to the Medical Records, Department of Ipswich Hospital for retrieving hospital records, and to Ms Leanne Tindale and Ms Ruth Archer for providing secretarial assistance. 
Medicine 2, 204-209.

Eisenberg M., Bergner L. \& Hallstrom A. (1979) Paramedic programmes and out-of-hospital cardiac arrests: 1. Factors associated with successful resuscitation. American Journal of Public Health 69, 30-38.

Eisenberg N. S., Hallstrom A. P., Copass N. K., et al. (1984) Treatment of ventricular fibrillation: emergency medical technician defibrillation and paramedic services. Journal of American Medical Association 251, 1723-1726.

Eisenberg M. S., Horwood B. T., Cummins R. O., et al. (1990) Cardiac arrest and resuscitation. A tale of 29 cities. Annals of Entergency Medicine, 179-186.

Hall J., Hawkins J. \& O'Rourke M. F. (1989) Pre-hospital cardiac arrest and its management in Sydney. Australia and Nere Zealand Journal of Medicine 19 (suppl 1), 538A.

Jacob I. \& Oxer H. (1990) A review of pre-hospital defibrillation by ambulance officers in Perth, Western Australia. Medical Journal of Australia 153, 662-664.

Pantridge J. F. \& Gettes J. S. (1967) A mobile intensive care unit in the management of myocardial infarction. Lancet 2, 271-273.

Sammel N. L., Taylor K., Selig M., et al. (1981) New South Wales intensive care ambulance assistance outcome of patients with ventricular fibrillation. Medical Journal of Australia 2, 546-550.

Stults K. R., Brown B. D., Schug B. L., et al. (1984) Pre-hospital defibrillation performed by emergency medical technicians in the rural community. New England Journal of Medicine 310, 219-223.

Weaver W. D., Cobb L. A., Hallstrom H. P., et al. (1986) Considerations for improving survival from out-of-hospital cardiac arrests. Annals of Emergency Medicine 15, 1181-1186.

Weaver W. D., Copass M. K., Buffi D., et al. (1984) Improved neurologic recovery and survival after early defibrillation. Circulation 69, 943-948. 